\title{
Teste anti-HIV na perspectiva das políticas públicas: proposta e realidade
}

\author{
HIV testing in the context of public policy: proposal and reality \\ Test anti - VIH en el contexto de las políticas públicas: propuesta y realidad
}

\author{
Elis Oliveira Arantes; Rosângela da Silva Santos ${ }^{I I}$
}

\begin{abstract}
RESUMO: Trata-se de um estudo de revisão integrativa realizado no Rio de Janeiro, com o objetivo de analisar a produção científica da área da saúde sobre a realização do teste contra o Vírus da Imunodeficiência Humana (HIV) na perspectiva das políticas públicas de saúde. Em relação aos quatro artigos selecionados neste estudo, apenas um deles discutiu o teste anti-HIV sob a ótica das políticas públicas. Os demais artigos abordaram o teste anti-HIV na atenção básica, sem mencionar as diretrizes políticas. Este fato evidencia que, apesar de existirem políticas públicas que estabelecem as ações de cuidados em relação ao teste anti-HIV, estas, além de não serem discutidas, não são implementadas nos serviços de saúde.

Palavras-Chave: HIV; mulheres; cuidados de enfermagem; atenção primária à saúde.
\end{abstract}

\begin{abstract}
This integrative review study, conducted in Rio de Janeiro, examined the healthcare-related scientific production regarding implementation of Human Immunodeficiency Virus (HIV) testing in the context of public health policies. Of the four articles selected in this study, only one discussed HIV testing from the public policy perspective. The remaining articles addressed HIV testing in primary care without, however, mentioning policy guidelines. This shows that, although there are public policies providing for health care actions in relation to HIV testing, not only are they not being discussed, they are not implemented in health services.
\end{abstract}

Keywords: HIV; women; nursing care; primary health care.

RESUMEN: Se trata de un estudio de revisión integradora realizado en Río de Janeiro, cuyo objetivo fue analizar la producción científica del área de salud sobre la aplicación del test del Virus de Inmunodeficiencia Humana (VIH) en el contexto de las políticas públicas de salud. Respecto con los cuatro artículos seleccionados en este estudio, sólo uno discutió el test anti-VIH desde la perspectiva de las políticas públicas. Los artículos restantes abordaron el test anti-VIH en la atención primaria sin mencionar, sin embargo, las directivas políticas. Este hecho demuestra que, si bien existen políticas públicas que establecen las acciones de cuidado en relación con el test anti-VIH, éstas no se discuten ni tampoco se ponen en marcha en los servicios de salud.

Palabras Clave: VIH; mujeres; atención de enfermería; atención primaria de salud.

\section{INTRODUÇÃO}

A Síndrome da Imunodeficiência Adquirida (AIDS) configura-se como um dos principais problemas de saúde pública no Brasil e no mundo devido a seu caráter evolutivo crônico e pandêmico que acomete mais de 35 milhões de pessoas no mundo, das quais 2,1 milhões se contaminaram em $2013^{1}$. Deste total, 17,6 milhões são mulheres em idade reprodutiva, representando mais de $50 \%$ do total de adultos infec$\operatorname{tados}^{1,2}$. Só na América Latina, são 550 mil mulheres infectadas pelo Vírus da Imunodeficiência Humana (HIV), sendo o Brasil responsável por 230.161 destas mulheres soropositivas ${ }^{1}$.

O Ministério da Saúde, em 2004, adotou como estratégia de prevenção e controle da infecção do HIV o acesso precoce ao teste anti-HIV para além dos Centros de Testagem Anônima (CTA), incluindo as unidades básicas de saúde (UBS), devendo ser oferecido a toda população que se encontra vulnerável à infecção, como por exemplo, os casos de Infecções Sexualmente Transmissíveis (IST) ${ }^{3-9}$. Tal medida justifica-se pelo fato de a taxa de transmissão ser 3,5 vezes maior no grupo de pessoas que desconhecem sua sorologia para o HIV?

Para a população, o diagnóstico precoce possibilita romper a cadeia de transmissão do HIV e favorece a instituição precoce do tratamento que permite maior qualidade de vida aos portadores do HIV e, consequentemente, menor morbimortalidade causada pela AIDS. Para a saúde pública, o diagnóstico oportuno diminui o custo relacionado às comorbidades da infecção pelo HIV e previne a transmissão deste vírus às pessoas soronegativas ${ }^{2,9,10,11}$.

\footnotetext{
${ }^{\mathrm{I}}$ Mestranda em Enfermagem pela Universidade do Estado do Rio de Janeiro. Faculdade de Enfermagem, Rio de Janeiro, Brasil. E-mail: elisarantes@outlook.com. IIProfessora Adjunta da Universidade do Estado do Rio de Janeiro. Faculdade de Enfermagem, Departamento Materno-Infantil. Rio de Janeiro, Brasil. Representante da área de Enfermagem no Conselho Assessor de Enfermagem do Conselho Nacional Pesquisa. E-mail: rosangelaufrj@gmail.com.
} 
Porém, apesar da descentralização dos testes anti-HIV e o incentivo para a testagem na população em geral, as mulheres que não vivenciam o período gestatório ainda se encontram à margem do diagnóstico precoce, mesmo com a duplicação do número de testes de HIV distribuídos e pagos pelo SUS, que passou de 3,3 milhões para 6,3 milhões de unidades ${ }^{2}$.

Portanto, refletir sobre a política de ampliação e descentralização do acesso ao teste anti-HIV faz-se necessário, uma vez que a população feminina sexualmente ativa e fora do período gestacional ainda possui um reduzido acesso às ações de aconselhamento e de diagnóstico para a infecção do HIV nas UBS. Esta baixa cobertura amplia a vulnerabilidade e os agravos relacionados à infecção, uma vez que o diagnóstico tardio posterga as ações educativas e de tratamento que visam oferecer qualidade de vida aos portadores do HIV e, consequentemente, diminuir a morbimortalidade ${ }^{9,12,13}$.

$O$ profissional de enfermagem tem respaldo legal e ético para que, quando devidamente treinado e capacitado de acordo com as normas do Ministério da Saúde, proceda à coleta, à leitura e ao registro do resultado do teste rápido anti-HIV ${ }^{14}$. Ademais, inseridos no Programa de Saúde da Família (PSF), estes profissionais criam vínculos com a população, o que favorece a realização do aconselhamento pré e pós-teste, bem como maior oportunidade em instituir educação sexual, planejamento familiar e uma assistência integral a todos os ciclos de vida da mulher ${ }^{3}$.

Esta pesquisa oferecerá subsídios para a realização de novos estudos e intervenções no campo da saúde das mulheres, especificamente, no que tange aos cuidados de enfermagem, para que os cuidados referentes à saúde da mulher sejam legitimados em todos os ciclos de vida e, assim, ser possível dar visibilidade às mulheres que não vivenciam o momento da gestação.

Para isso, este estudo utilizou o método da revisão integrativa, a fim de identificar se a produção científica segue as recomendações previstas para a testagem anti-HIV. Para guiar a revisão integrativa, formulou-se a seguinte questão norteadora: $\mathrm{O}$ teste anti-HIV é disponibilizado para as mulheres fora do período gestacional durante o exame ginecológico nas UBS? O objetivo foi analisar a produção científica da área da saúde em relação à realização do teste antiHIV, na perspectiva das políticas públicas de saúde.

\section{Metodologia}

Este estudo utilizou o método de revisão integrativa, que inclui a análise profunda de pesquisas relevantes e sintetiza, de maneira sistemática e ordenada, os resultados de diversos estudos sobre uma determinada temática ${ }^{15}$. Utilizou-se como técnica de pesquisa um formulário de coleta de dados elaborado pelas autoras contendo perguntas fechadas.
A construção desta revisão integrativa contemplou as seis fases propostas no método: seleção da questão norteadora, seleção das pesquisas que constituem a amostra do estudo, representação das características das pesquisas revisadas, análise dos achados de acordo com os critérios de inclusão e exclusão estabelecidos, interpretação e divulgação dos resultados ${ }^{16,17}$.

Os critérios de inclusão dos artigos selecionados foram: abordar o teste anti-HIV; texto completo disponível na Biblioteca Virtual em Saúde (BVS) e na Science Direct e ano de publicação de 2004 a 2013, período em que entra em vigor a política de ampliação e descentralização dos testes anti-HIV nas UBS. O critério de exclusão foi abordar o período gestacional.

A busca dos artigos ocorreu nos meses de novembro e dezembro de 2014 na BVS empregando-se os descritores: HIV, mulheres e enfermagem. A princípio, cada descritor foi utilizado individualmente, sem adotar filtro e, posteriormente, com os filtros: texto completo disponível e ano de publicação - 2004 a 2013. Em seguida, foram usados os mesmos descritores, porém conjugados, primeiramente sem utilização dos filtros e, em seguida, com eles.

No levantamento da Science Direct, os mesmos descritores citados foram adotados, porém no idioma inglês: HIV, womens e nursing e também com os mesmos filtros: texto completo disponível e ano de publicação de 2004 a 2013. Cada descritor foi empregado individualmente, sem adotar filtro e, posteriormente, com filtro. Em seguida, foram utilizados os mesmos descritores, porém, conjugados, primeiramente sem o uso dos filtros e, em seguida, com eles.

Ao conjugar os descritores e utilizar os filtros, remeteu-se ao seguinte quantitativo: 90 artigos na Science Direct; 46 artigos no Lilacs; 24 artigos na Medline e 11 artigos na Scielo. Ao realizar a leitura na íntegra destes textos, apenas quatro definiram a amostra final da presente revisão: dois na Science Direct, um na Medline e um no Lilacs.

Para avaliação crítica, realizou-se a leitura na íntegra destes estudos e a releitura dos resultados, com finalidade de identificar aspectos relevantes que se repetiam ou se destacavam. Em seguida, os artigos foram identificados por números, de acordo com a ordem de localização e foram catalogados em ficha bibliográfica adaptada e organizada da seguinte forma: título, autores, formação, ano, país, idioma, periódico, nível de evidência, objetivos, participantes, resultados e conclusões.

Os resultados foram discutidos com a literatura pertinente ao tema ${ }^{3-5,9-14,18-30}$.

\section{Resultados e Discussão}

Em relação à caracterização dos quatro artigos selecionados, dois foram publicados em 2008 (A1 e A2) 22,26 $^{2}$ e os outros dois (A3 e A4) $)^{24,25} \mathrm{em} 2013$. Somente um era proveniente de pesquisa brasileira $(\mathrm{A} 2)^{26}$, dois do con- 
tinente africano $(\mathrm{A} 3 \mathrm{e} \mathrm{A} 4)^{24,25}$ e um americano (A1) ${ }^{22}$. Quanto à formação dos autores, um dos artigos (A1) 22 teve como pesquisadores profissionais de enfermagem e de medicina, outro (A2) ${ }^{26}$, apenas profissionais de enfermagem e dois artigos (A3 e A4) $)^{24,25}$ tiveram como pesquisadores profissionais médicos. Ver Figura 1.

$\mathrm{O}$ artigo $\mathrm{A} 1^{22}$ verificou as percepções de mulheres que frequentam unidades básicas de saúde em relação às novas recomendações para realização do teste anti-HIV nos EUA. $\mathrm{O}$ artigo A2 ${ }^{26}$ enfatizou as questões do sigilo do diagnóstico na assistência do PSF; A3 verificou os motivos que levaram a alta aceitação do teste anti-HIV quando realizado no domicílio e $\mathrm{A} 4{ }^{25}$, que consistiu em um desdobramento do artigo $\mathrm{A} 3^{24}$, buscou avaliar a questão do acesso e os efeitos negativos da realização do teste anti-HIV no domicílio.

A avaliação da qualidade das evidências científicas dos estudos em questão teve como objetivo determinar a confiabilidade dos estudos na utilização dos resultados e fortalecer as conclusões que irão gerar o estado do conhecimento atual do tema investigado. Assim, um artigo foi classificado como nível de evidência I (A4) ${ }^{25}$, por se tratar de ensaio clínico randomizado e os outros três $\left(\mathrm{A} 1^{22}, \mathrm{~A} 2^{26}\right.$ e $\left.\mathrm{A} 3^{24}\right)$ foram classificados como nível de evidência IV, por utilizarem o método qualitativo descritivo ${ }^{17}$.

$\mathrm{O}$ artigo $\mathrm{A} 44^{25}$ aborda a realização do aconselhamento e do teste anti-HIV nas mulheres juntamente com seus parceiros como ponto positivo para aceitação do teste. De acordo com as mulheres do estudo, o envolvimento do parceiro na realização do teste as isenta da responsabilidade em revelar o diagnóstico e recrutar seus maridos para fazê-lo, quando indicado pelo profissional de saúde.
Vale resgatar que o estigma e o preconceito relacionados à infecção são provenientes do início da epidemia, que se alastrou rapidamente pelo mundo e pouco se sabia a seu respeito. Até 1984, quatro anos após o primeiro caso de infecção pelo HIV no mundo, a AIDS ficou conhecida como Peste Gay e associada aos grupos denominados de risco, como os homossexuais, hemofílicos, profissionais do sexo e usuários de drogas injetáveis ${ }^{18}$. A urgência de resposta à epidemia, o pouco conhecimento relacionado a ela e a concentração de casos transgressores dos bons costumes da sociedade geraram políticas moralistas que espalharam e perpetuaram o preconceito e o estigma ao $\mathrm{HIV}^{19,20}$.

A infecção pelo HIV no Brasil e no mundo evidenciou contradições sociais, econômicas e culturais, que desafiaram a busca por respostas e pelo estabelecimento de políticas de saúde para o controle da epidemia ${ }^{21,22}$. O pouco conhecimento sobre essa nova epidemia que acometia o mundo, em 1980, trouxe como consequências o preconceito e o estigma, que ainda se perpetuam sobre o HIV e se constituem em importantes barreiras para a implementação das políticas públicas voltadas para a infecção ${ }^{19}$.

Apesar de estas políticas brasileiras representarem um nível de excelência e terem, muitas delas, o reconhecimento internacional, ainda apresentam grandes desafios em sua execução ${ }^{19,22}$. É o que acontece com as políticas e programas sobre o teste anti-HIV que são bastante limitadas, devido aos diversos fatores que influenciam na decisão de aceitar ou não o teste, por sofrerem ainda influências das medidas preconceituosas e excludentes adotadas no início da epidemia.

\begin{tabular}{|c|c|c|c|}
\hline Título/Autores & Periódico & País / Idioma & Participantes \\
\hline $\begin{array}{l}\text { A1. The Centers for Disease } \\
\text { Control and Prevention revised } \\
\text { recommendations for HIV testing: } \\
\text { reactions of women attending } \\
\text { community health clinics. }{ }^{22}\end{array}$ & $\begin{array}{c}\text { J Assoc } \\
\text { Nurses AIDS } \\
\text { Care } 2008\end{array}$ & EUA / Inglês & $\begin{array}{l}30 \text { mulheres que } \\
\text { frequentam três clínicas } \\
\text { comunitárias - ambiente } \\
\text { primário de saúde. }\end{array}$ \\
\hline $\begin{array}{l}\text { A2. A Abertura da privacidade e o } \\
\text { sigilo das informações sobre o } \\
\text { HIV/Aids das mulheres atendidas } \\
\text { pelo programa saúde da família no } \\
\text { município de São Paulo, Brasil }{ }^{26}\end{array}$ & $\begin{array}{c}\text { Saude soc. } \\
2008\end{array}$ & $\begin{array}{c}\text { Brasil / } \\
\text { Português }\end{array}$ & $\begin{array}{l}5 \text { Médicos. } 6 \\
\text { enfermeiras. } 14 \text { auxiliares } \\
\text { de enfermagem e agentes } \\
\text { comunitários de saúde } \\
\text { (ACS). }\end{array}$ \\
\hline $\begin{array}{l}\text { A3. The seven Cs of the high } \\
\text { acceptability of home-based VCT: } \\
\text { results from a mixed methods } \\
\text { approach in Zambia }{ }^{24}\end{array}$ & $\begin{array}{c}\text { Social } \\
\text { Science \& } \\
\text { Medicine } \\
2013\end{array}$ & $\begin{array}{l}\text { Zambia / } \\
\text { Inglês }\end{array}$ & $\begin{array}{l}836 \text { adultos - grupo de } \\
\text { intervenção e } 858 \text { adultos } \\
\text { - grupo controle. } 1.501 \\
\text { pessoas pesquisadas em } \\
36 \text { aldeias / Zâmbia. }\end{array}$ \\
\hline $\begin{array}{l}\text { A4. Strong effects of home-based } \\
\text { voluntary HIV counselling and } \\
\text { testing on acceptance and equity: a } \\
\text { cluster randomised trial in } \\
\text { Zambia }^{25}\end{array}$ & $\begin{array}{l}\text { Social } \\
\text { Science \& } \\
\text { Medicine } \\
2013\end{array}$ & $\begin{array}{l}\text { Zambia / } \\
\text { Inglês }\end{array}$ & $\begin{array}{l}836 \text { adultos - grupo de } \\
\text { intervenção e } 858 \text { adultos } \\
\text { - grupo controle. } 1.501 \\
\text { pessoas pesquisadas em } \\
36 \text { aldeias / Zâmbia. }\end{array}$ \\
\hline
\end{tabular}

Fonte: Science Direct, Lilacs, e Medline

FIGURA 1: Identificação dos artigos selecionados para o estudo. 
Ademais, quando estas políticas estão voltadas para as mulheres, os desafios são ainda maiores, já que durante décadas as mulheres foram vistas apenas para gerar filhos, sem direito de vivenciarem a sexualidade da forma que desejassem, pois recaíam sobre elas as questões de gênero ${ }^{21}$. Mesmo com programas do governo assegurando os direitos sexuais e reprodutivos das mulheres e com a Política Nacional de Atenção Integral à Saúde da Mulher, em 2004, as políticas públicas ainda priorizam a população feminina apenas no período gestacional, principalmente no que tange ao $\mathrm{HIV}^{3}$.

No início do ano de 2000, as políticas públicas brasileiras iniciaram o processo de ampliação do teste anti-HIV para além dos Centros de Testagem e Aconselhamento (CTA), introduzindo-o na UBS, independente das consultas de pré-natal. Esta medida ganhou força com o Projeto AIDS III e com a Campanha Fique Sabendo que, entre outros objetivos, destacaram a ampliação do acesso ao diagnóstico precoce do HIV e a melhoria da qualidade da atenção às DST/AIDS ${ }^{19,22}$.

Em 2004, o governo brasileiro efetivou a oferta do teste rápido para o HIV, nas UBS, para a população em geral, com o intuito de ampliar e descentralizar a aplicação do teste anti-HIV, que antes estava concentrado nos CTA e fora dele, somente para gestantes ${ }^{21}$. Assim como o Brasil, os Estados Unidos também propuseram a ampliação do teste anti-HIV para a população em geral, mas somente a partir de $2006^{22}$. A proposta trouxe o fim do serviço de aconselhamento pré e pós-teste, o fim do consentimento por escrito para a realização do teste anti-HIV e a oferta do teste para a população na faixa etária dos 13 aos 69 anos ${ }^{23,24}$.

Porém, nota-se, muitas vezes, que o teste antiHIV está sendo realizado somente durante a gestação e de forma compulsória, sem aconselhamento pré e pós-teste, sem que as mulheres saibam que o exame é para sorologia do $\mathrm{HIV}^{25-27}$. A frivolidade do teste anti-HIV, nas consultas de pré-natal, demonstra que os profissionais de saúde estão mais preocupados em cumprir protocolos e metas do que zelar pela saúde e pela vida de mãe e filho ${ }^{24-26}$.

A preocupação em conhecer a opinião das mulheres em relação a como e quando deve ser realizado o teste anti-HIV é notória na produção científica, porém, na prática profissional, essa preocupação, autonomia e evidência não são dadas às mulheres para que elas façam escolhas conscientes em relação a aceitar ou não a realização do teste anti-HIV em qualquer ciclo de suas vidas ${ }^{22-26}$. Os profissionais de saúde ainda trabalham com o conceito de grupos de risco e, sobretudo, incorporam as questões de gênero, que distanciam as mulheres que não se enquadram nestes grupos das ações educativas e preventivas à saúde ${ }^{28-30}$.

A produção científica examinada revelou que o teste anti-HIV, quando realizado em domicílio, proporciona às mulheres maior confiabilidade no sigilo profissional em relação ao diagnóstico. $O$ fato de irem até à UBS e serem testadas para o HIV, independente do diagnóstico, já predispõe ao estigma e à discriminação, como disse uma mulher do estudo Os olhos das pessoas podem matar vocế ${ }^{25}$. Ademais, o teste oferecido em domicílio tem potencial para diminuir as diferenças sociais e as desigualdades de gênero no acesso ao teste anti-HIV que surgiram devido ao aumento de gestantes testadas para o HIV ${ }^{22,25}$.

Outro ponto evidenciado é que os profissionais de saúde inseridos no PSF reconhecem o papel importante dos agentes comunitários de saúde por estarem inseridos na comunidade e, portanto, reconhecem as mulheres em situações de risco para o HIV. Por isso, discutir a importância do diagnóstico precoce entre os profissionais da equipe é fundamental, para que, juntos, possam facilitar o acesso ao teste anti-HIV e traçarem um plano de cuidados às portadoras do HIV ${ }^{26}$.

A realização do aconselhamento e do teste antiHIV nas mulheres, juntamente com seus parceiros, foi um aspecto positivo para aceitação do teste por parte das mulheres, segundo a produção científica analisada $^{24,25}$. De acordo com as mulheres, serem testadas em casa e junto com seus parceiros diminui a responsabilidade por não serem as únicas a conhecer seu estado sorológico para o HIV e as isentam da responsabilidade de recrutarem seus parceiros para realização do teste ${ }^{24}$.

\section{Conclusão}

Em relação aos quatro artigos selecionados neste estudo, apenas um discute o teste anti-HIV sob a ótica das políticas públicas. Os demais artigos abordam o teste anti-HIV na atenção básica sem mencionar as diretrizes políticas ${ }^{14,15,16,18}$. Este fato evidencia que, apesar de existirem políticas públicas que estabeleçam as ações de cuidados em relação ao teste anti-HIV, estas, além de não serem discutidas, não são implementadas nos serviços de saúde.

Mesmo com a política de ampliação e descentralização do teste anti-HIV para a população em geral, seus propósitos não têm sido totalmente atingidos. As mulheres que não vivenciam e nem desejam vivenciar a gestação continuam invisíveis no que tange ao diagnóstico precoce do HIV, pois os profissionais de saúde inseridos nas UBS ainda direcionam os testes somente para as consultas de pré-natal e para os ultrapassados de grupos de risco, o que perpetua o estigma e o preconceito relacionados ao teste anti-HIV.

É notório que, assim como na prática das UBS, a produção científica examinada destaca os testes anti-HIV apenas para as consultas de pré-natal, sem nenhuma menção da oferta destes testes nas consultas ginecológicas.

É necessário abordar a realização do teste anti-HIV nas consultas ginecológicas e evidenciar a 
política nacional de ampliação do teste anti-HIV, já que é um direito de cidadania o acesso aos serviços de saúde pública e o conhecimento precoce da sorologia para o HIV. É preciso que a prática profissional, no que tange ao teste anti-HIV em mulheres, aconteça de forma integral e com equidade, sem condicioná-lo somente ao período gestacional.

\section{REFERÊNCIAS}

1.United Nations Programme On HIV/AIDS. 2014 report on the global HIV/AIDS epidemic: 4th global report. Geneva: UNAIDS, 2014. [cited in 2015 July 10] Disponível em: http://www.unaids.org/en/resources/presscentre/pressreleaseandstatementarchive/2014/ july/2010716prgapreport.

2.Ministério da Saúde (Br). Secretaria de Assistência à Saúde. Boletim Epidemiológico - Aids e DST. Guia de Vigilância Epidemiológica. Brasília (DF): Ministério da Saúde; 2013.

3.Ministério da Saúde (Br). Secretaria de Vigilância em Saúde. Departamento de DST, aids e Hepatites Virais. Cadernos de boas práticas em HIV/Aids na Atenção Básica. Brasília (DF): Ministério da Saúde; 2014.

4.Denize Z. Aconselhamento pré e pós-teste anti-HIV na atenção básica: as perspectivas dos profissionais de saúde [dissertação de mestrado]. São Paulo: Universidade de São Paulo; 2012.

5.Araújo CLF, Priscila AS, Gleice KAS, Maíra GPO, Livia SC. A testagem anti-HIV nos serviços de ginecologia do município do Rio de Janeiro. Esc Anna Nery. 2014; 18:82-9. 6.Mariângela GS, Francisco IB. HIV/aids no Brasil: provimento de prevenção em um sistema descentralizado de saúde. Questões relativas à provisão universal de saúde. GHD-018. 7.Szwarcwald CL, Castilho EA. A epidemia de HIV/AIDS no Brasil: três décadas. Cad Saúde Pública. 2011; 27 (suppl 1):4-5. 8.Ministério da Saúde (Br). Boletim epidemiológico 2013 DST/AIDS. Brasília (DF): Ministério da Saúde; 2013.

9.Alejandra XA. Facilitadores y barreras que enfrentan las personas al tomar el test de ELISA para el diagnóstico del VIH: revisión de la literatura. Rev Chilena Infectol. 2013; 30: 638-43. 10.Pereira BS. Enfrentamento da feminização da epidemia da AIDS. FG Ciência. 2011; 1(1):118.

11.Moreschi C. Mulheres e vulnerabilidade ao HIV/ AIDS. Saúde Santa Maria. 2012; 38(2):85-94.

12.Leonardo GF, Richardson ARS, Ilisdayne TSS, Karla GSS, Francisca FAS. Percepção de mulheres casadas sobre o risco de infecção pelo hiv e o comportamento preventivo. Rev enferm UERJ. 2013; 21:805-11.

13.Aboim S. Risco e prevenção do HIV/AIDS: uma perspectiva biográfica sobre os comportamentos sexuais em Portugal. Ciênc saúde coletiva. [Scielo- Scientific Electronic Library Online] 2012 [citado em 12 out 2014]. 17:99-112. Disponível em: http://www.scielosp.org/pdf/csc/v17n1/a13v17n1.pdf.

14.Conselho Federal de Enfermagem. Parecer Normativo $n^{0}$ 001/2013. Legislação profissional. Competência do enfermeiro para realizar teste rápido para detecção de HIV, sífilis e outros agravos. [citado em 10 jun 2015] Disponível em: http://novo.portalcofen.gov.br/parecernormativo-no-0012013_18099.html.
15.Mendes KDS, Silveira RCCP, Galvão CM. Revisão Integrativa: método de pesquisa para a incorporação de evidências na saúde e na enfermagem. Texto contexto - enferm. 2008; 17:758-64.

16.Souza MT, Silva MD, Carvalho R. Revisão Integrativa: como é e como fazer. Einsten. 2010; 8(1):102-6.

17.Polit DF, Beck CT. Fundamentos de pesquisa em enfermagem: avaliação de evidências para a prática de enfermagem. $7^{\mathrm{a}}$ ed. Porto Alegre (RS): Artmed; 2011. 18.Cau BM. O estigma do HIV e SIDA e a testagem de infecção pelo HIV entre adolescentes e jovens em Moçambique. Gazeta de População e Saúde. 2014; 3(4):1-33. 19.Villarinho MV. Políticas públicas de saúde face à epidemia da AIDS e a assistência às pessoas com a doença. Rev Bras Enferm. 2013; 66:271-7.

20.Rodrigues CS, Perreault M. A feminização do HIV/ AIDS sob a perspectiva de mulheres infectadas: uma questão de gênero. In: III Seminário Internacional Enlaçando Sexualidades; 2013 mai 15-17; Salvador, Brasil. Salvador (BA): Universidade do Estado da Bahia; 2013. 21.Ministério da Saúde (Br). Secretaria de Atenção à Saúde Departamento de Ações Programáticas Estratégicas. Política Nacional de Atenção Integral à Saúde da Mulher - Princípios e Diretrizes. Brasília (DF): Editora MS; 2011. 22.Burrage JW, Zimet GD, Cox DS, Cox AD, Mays RM, Fife RS, et al. The Centers for Disease Control and Prevention revised recommendations for HIV testing: reactions of women attending community health clinics. J Assoc Nurses AIDS Care. 2008; 19:66-74.

23.Pimenta C, Raxach JC, Terto Júnior V, organizadores. Prevenção das DST/AIDS e novos desafios. Rio de Janeiro: Abia; 2010.

24.Jürgensen M, Sandøy IF, Michelo C, Fylkesnes K, Mwangala S, Blystad A. The seven $\mathrm{C}$ sof the high acceptability of home-based VCT: Results from a mixed methods approach in Zambia. Social Science \& Medicine. 2013; 97:210-219.

25.Fylkesnes K, Sandøy IF, Jürgensen M, Chipimo PJ, Mwangala S, MicheloC. Strong effects of home-based voluntary HIV counseling and testing on acceptance and equity: A cluster randomized trial in Zambia. Social Science \& Medicine. 2013; 86:9-16.

26.Abdalla FTM, Nichiata LYI. A abertura da privacidade e o sigilo das informações sobre o HIV/Aids das mulheres atendidas pelo Programa Saúde da Família no Município de São Paulo, Brasil. Saúde Soc. 2008; 17(2):140-52.

27.Feitosa JÁ, Coriolano MWL, Alencar EM, Lima LS. Aconselhamento do pré-teste anti-HIV no pré-natal: percepções da gestante. Rev enferm UERJ. 2010;18:559-64. 28.Bastos DC, Paiva MS, Carvalho ESS, Rodrigues GRS. Representações sociais da vulnerabilidade de mulheres negras e não negras à infecção pelo HIV/AIDS. Rev enferm UERJ. 2013; 21:330-6.

29. Galvão MTG, Gouveia AS, Carvalho CML, Costa Ê, Freitas JG, Lima ICV. Temáticas produzidas por portadores de HIV/AIDS em grupo de autoajuda. Rev enferm UERJ. 2011; 19:299-304.

30.Branheto ACM, Carvalho AMP. Narrativa da mulher, mãe infectada pelo HIV. Saúde \& Transformação Social. 2013; 4:47-52. 